\title{
Persymmetric Parametric Adaptive Matched Filter for Multichannel Adaptive Signal Detection
}

Wang, P.; Sahinoglu, Z.; Pun, M-O.; Li, H.

TR2012-031 March 2012

\begin{abstract}
This paper considers a parametric approach for multichannel adaptive signal detection in Gaussian disturbance which can be modeled as a multichannel auto-regressive (AR) process and, moreover, possesses a persymmetric structure induced by a symmetric antenna geometry. By introducing the persymmetric AR (PAR) modeling for the disturbance, a persymmetric parametric adaptive matched filter (Per-PAMF) is proposed. The developed Per-PAMF extends the classical PAMF by exploiting the underlying persymmetric properties and, hence, improves the detection performance in training-limited scenarios. The performance of the proposed Per-PAMF is examined by the Monte-Carlo simulations and simulation results demonstrate the effectiveness of the Per-PAMF compared with the conventional PAMF and non-parametric detectors.
\end{abstract}

IEEE Transactions on Signal Processing

This work may not be copied or reproduced in whole or in part for any commercial purpose. Permission to copy in whole or in part without payment of fee is granted for nonprofit educational and research purposes provided that all such whole or partial copies include the following: a notice that such copying is by permission of Mitsubishi Electric Research Laboratories, Inc.; an acknowledgment of the authors and individual contributions to the work; and all applicable portions of the copyright notice. Copying, reproduction, or republishing for any other purpose shall require a license with payment of fee to Mitsubishi Electric Research Laboratories, Inc. All rights reserved. 



\section{Persymmetric Parametric Adaptive Matched Filter for Multichannel Adaptive Signal Detection}

Pu Wang, Zafer Sahinoglu, Man-On Pun, and Hongbin Li

\begin{abstract}
This paper considers a parametric approach for multichannel adaptive signal detection in Gaussian disturbance which can be modeled as a multichannel auto-regressive (AR) process and, moreover, possesses a persymmetric structure induced by a symmetric antenna geometry. By introducing the persymmetric AR (PAR) modeling for the disturbance, a persymmetric parametric adaptive matched filter (PerPAMF) is proposed. The developed Per-PAMF extends the classical PAMF by exploiting the underlying persymmetric properties and, hence, improves the detection performance in training-limited scenarios. The performance of the proposed Per-PAMF is examined by the Monte-Carlo simulations and simulation results demonstrate the effectiveness of the Per-PAMF compared with the conventional PAMF and non-parametric detectors.
\end{abstract}

Index Terms-Multichannel adaptive signal detection, multichannel auto-regressive process, parametric approach, persymmetry, maximum likelihood estimation.

\section{INTRODUCTION}

Multichannel adaptive signal detection against strong spatially and temporally colored disturbances has been encountered in many applications, e.g., wireless communications, hyperspectral imaging, and medical imaging [1]-[3]. Traditional techniques are limited for practical applications due to their excessive training requirement and high computational complexity. For example, the covariance-matrixbased detectors, e.g., Kelly's generalized likelihood ratio test (GLRT) [4], the adaptive matched filter (AMF) [5] and the recent Rao test [6], need $K \geq J N$ training signals to ensure a full-rank estimate of the disturbance covariance matrix and have to invert the $J N \times J N$ covariance matrix, where $J$ denotes the number of antennas and $N$ denotes the number of pulses.

Among other techniques, a class of parametric detectors provide an efficient way to simultaneously mitigate the training requirement and reduce the computational complexity [7]-[11] (and reference therein). By modeling the disturbance as a multichannel auto-regressive (AR) process, the parametric detectors decompose the jointly spatio-temporal whitening of the covariance-matrix-based detectors into successive temporal whitening and spatial whitening. As a well-known parametric detector, the parametric AMF (PAMF) is simple to implement. Using measured datasets [7], [8], the PAMF was found to yield better performance with significantly reduced computational complexity than the non-parametric counterpart, i.e., the AMF, especially when $K \ll J N$.

In this paper, extending the multichannel AR process based parametric detector, we exploit additional structure of the disturbance covariance matrix, i.e., the persymmetric property [12], [13]. This enables an improved parametric detector with better training-signal efficiency. The utilization of persymmetry for applications in communications and radar can be traced back to [12], [13] and has been proved to be an efficient way to mitigate the demanding requirement of homogeneous training signals. In [13], Nitzberg shows that the efficiency of usage of training signals is improved by up to a factor of two by utilizing persymmetry. Following [13], several adaptive detection schemes explicitly taking into account the persymmetry

P. Wang and $\mathrm{H}$. Li are with the Department of Electrical and Computer Engineering, Stevens Institute of Technology, Hoboken, NJ 07030, USA (email: \{pwang4, Hongbin.Li\} @ stevens.edu).

Z. Sahinoglu and M. O. Pun are with Mitsubishi Electric Research Laboratories (MERL), 201 Broadway, Cambridge, MA 02139, USA (e-mail: \{zafer, mpun\}@merl.com). have been proposed in [14] and, more recently, [15]-[19]. Specifically, [14] proposed a GLRT to detect a multi-band signal from the disturbance with the persymmetric property. Extension to the compound-Gaussian environment was made in [15] and has been further verified in [16] with experimentally measured datasets. The results show that exploiting the persymmetric property significantly improves the robustness of the adaptive detection algorithms in terms of the constant false alarm rate (CFAR). Meanwhile, [17] proposed a persymmetric GLRT for a partially-homogeneous environment.

Our incorporation of the persymmetric constraint in the PAMF leads to a new persymmetric PAMF (Per-PAMF), which, while maintaining the simple implementation as the PAMF, further improves the robustness in training-limited scenarios. The Per-PAMF is developed in a two-step procedure. In detail, a non-adaptive parametric matched filter (PMF) is first introduced by assuming the knowledge of the nuisance parameters and, then, the Per-PAMF is developed from the PMF by replacing the nuisance parameters by their maximum likelihood (ML) estimates from training signals under the persymmetric constraint. The performance of the Per-PAMF detector is examined by the Monte-Carlo simulations and the simulation results show that the Per-PAMF has slightly better performance than the PAMF when the number of training signals is sufficient, while it significantly outperforms the the PAMF in cases with extremely limited training signals.

The remainder of the paper is organized as follows. Section II contains the signal model and introduces the persymmetric AR modeling for the disturbance. The Per-PAMF detector is derived in Section III. Numerical results with two distinct datasets are provided in Section IV. The conclusion is finally drawn in Section V.

\section{Signal Model}

The problem of interest is to decide which of the following two hypotheses is true [4], [5], [7], [9], [10]:

$$
\begin{aligned}
& H_{0}: \mathbf{x}_{0}=\mathbf{d}_{0}, \\
& H_{1}: \mathbf{x}_{0}=\alpha \mathbf{s}+\mathbf{d}_{0},
\end{aligned}
$$

where $\mathbf{x}_{0}$ is the $J N \times 1$ test signal, $\mathbf{s}$ is the known space-time steering vector which is a Kronecker product between the temporal $\left(\mathbf{s}_{d}\right)$ and spatial ( $\left.\mathbf{s}_{s}\right)$ steering vectors, i.e., $\mathbf{s}=\mathbf{s}_{d} \otimes \mathbf{s}_{s}, \alpha$ is an unknown complex-valued amplitude, and $\mathbf{d}_{0}$ is the disturbance signal (e.g., clutter and noise) which is modeled as a complex Gaussian vector with zero-mean and unknown covariance matrix $\mathbf{R}$, i.e., $\mathbf{d}_{0} \sim \mathcal{C} \mathcal{N}(\mathbf{0}, \mathbf{R})$. Aside from the test signal, there are $K$ targetfree independent and identically distributed (i.i.d.) training signals $\mathbf{x}_{k}=\mathbf{d}_{k} \sim \mathcal{C} \mathcal{N}(\mathbf{0}, \mathbf{R}), k=1, \cdots, K$, which are also independent of the test signal.

Moreover, by following the parametric approach introduced in [7], the disturbance signals $\mathbf{d}_{k}$ are modeled as a multichannel AR process. Specifically, let $\mathbf{d}_{k}(n) \in \mathbb{C}^{J \times 1}, n=0,1, \ldots, N-1$, denote the $N$ non-overlapping temporal segments of $\mathbf{d}_{k}$, i.e., $\mathbf{d}_{k} \triangleq$ $\left[\mathbf{d}_{k}^{T}(0), \mathbf{d}_{k}^{T}(1), \cdots, \mathbf{d}_{k}^{T}(N-1)\right]^{T}$. The multichannel AR process of the disturbance signal is described as [7], [9], [10]

$$
\mathbf{d}_{k}(n)=-\sum_{p=1}^{P} \mathbf{A}^{H}(p) \mathbf{d}_{k}(n-p)+\varepsilon_{k}(n),
$$

where $\varepsilon_{k}(n) \sim \mathcal{C N}(0, \mathbf{Q})$ is the $J$-channel temporally white but spatially colored Gaussian driving noise with $\mathbf{Q}$ denoting the unknown $J \times J$ spatial covariance matrix, and $\{\mathbf{A}(p)\}_{p=1}^{P}$ denote the unknown $J \times J$ AR coefficient matrices.

In this paper, we consider a case frequently encountered in practice, where the systems use a symmetric antenna configuration (symmetrical with respect to its phase center) and transmit a set of 
pulses of equal duration [13]-[17], [19]. For example, the widely used uniform linear array with a constant pulse repetition frequency (PRF) is such a system. The structured antenna array configurations and constant PRF cause the spatio-temporal covariance matrix $\mathbf{R}$ to be persymmetric-block-Toeplitz, as shown in [20]. Specifically, the return from a discrete disturbance source has a similar form as a target echo. Unlike a target, the disturbance is distributed in both range and azimuth. As an approximation to a continuous field, the disturbance return from a specific range is modeled as the superposition of a large number $N_{c}$ of independent disturbance sources in azimuth. Assuming that returns from different disturbance sources are uncorrelated, the disturbance covariance matrix can be calculated as [20, Section 2.6.1]

$$
\mathbf{R}=\sum_{i=1}^{N_{c}} \epsilon_{i}\left(\mathbf{s}_{d, i} \mathbf{s}_{d, i}^{H}\right) \otimes\left(\mathbf{s}_{s, i} \mathbf{s}_{s, i}^{H}\right),
$$

where $\epsilon_{i}$ is proportional to the radar cross section (RCS) for the $i$-th disturbance source, and $\mathbf{s}_{d, i}$ and $\mathbf{s}_{s, i}$ denote the temporal (Doppler) and spatial steering vectors, respectively. Due to the configurations in both spatial and temporal domains, the temporal and spatial steering vectors of the disturbance source satisfy the following properties, i.e., $\mathbf{s}_{d, i}$ is a Vandermonde vector and $\mathbf{s}_{s, i}=\mathbf{E} \mathbf{s}_{s, i}^{*}$ is persymmetric, where $\mathbf{E}$ denotes the exchange matrix with unit anti-diagonal elements and zeros elsewhere,

$$
\mathbf{E}=\left[\begin{array}{ccccc}
0 & 0 & \cdots & 0 & 1 \\
0 & 0 & \cdots & 1 & 0 \\
\vdots & \vdots & . & \vdots & \vdots \\
0 & 1 & \cdots & 0 & 0 \\
1 & 0 & \cdots & 0 & 0
\end{array}\right]
$$

Therefore, $\mathbf{s}_{d, i} \mathbf{s}_{d, i}^{H}$ is a Toeplitz matrix, while $\mathbf{s}_{s, i} \mathbf{s}_{s, i}^{H}$ is a persymmetric matrix. In addition to the Kronecker operation in (3), it is straightforward to show that the overall covariance matrix $\mathbf{R}$ to be a persymmetric-block-Toeplitz matrix.

To exploit this structure information of the covariance matrix $\mathbf{R}$, a new multichannel AR model by incorporating the persymmetric property is introduced below.

- AS1 - Persymmetric Spatial Covariance Matrix: Following the traditional AR process of (2), the spatial covariance matrix is further assumed to be persymmetric as

$$
\mathbf{Q}=\mathbf{E Q}^{*} \mathbf{E},
$$

where $[\cdot]^{*}$ denotes the complex conjugate and $\mathbf{E}$ denotes the exchange matrix of (4).

- AS2 - Persymmetric AR Coefficient Matrices: In addition to the persymmetric $\mathbf{Q}$, we assume that the AR coefficient matrices satisfy the following property

$$
\mathbf{A}(p)=\mathbf{E A}^{*}(p) \mathbf{E} .
$$

As shown in the Appendix, we prove that the proposed persymmetric AR model provides a parametric approach to approximate the persymmetric-block-Toeplitz structure of the covariance matrix $\mathbf{R}$. In summary, the goal is to develop a decision rule for the problem in (1) together with the assumptions AS1 and AS2.

\section{Persymmetric Parametric Adaptive Matched Filter}

The Per-PAMF is developed in a two-step approach: 1) find the GLRT when the nuisance parameters $\mathbf{A}$ and $\mathbf{Q}$ are assumed both known; 2) replace $\mathbf{A}$ and $\mathbf{Q}$ by their $\mathrm{ML}$ estimates from training signals subject to the persymmetric constraints.

\section{A. PMF - the GLRT with Known $\mathbf{A}$ and $\mathbf{Q}$}

When $\mathbf{A}$ and $\mathbf{Q}$ are both known, the GLRT has the form as

$$
T=\frac{\max _{\alpha} p_{1}\left(\mathbf{x}_{0} ; \alpha, \mathbf{A}, \mathbf{Q}\right)}{p_{0}\left(\mathbf{x}_{0} ; \mathbf{A}, \mathbf{Q}\right)},
$$

where $\left\{p_{i}\right\}_{i=0,1}$ are the likelihood functions under $H_{0}$ and $H_{1}$. It can easily be shown that the GLRT reduces to the non-adaptive PMF [7]

$$
T_{\mathrm{PMF}}=\frac{\left|\sum_{n=P}^{N-1} \tilde{\mathbf{s}}^{H}(n) \mathbf{Q}^{-1} \tilde{\mathbf{x}}_{0}(n)\right|^{2}}{\sum_{n=P}^{N-1} \tilde{\mathbf{s}}^{H}(n) \mathbf{Q}^{-1} \tilde{\mathbf{s}}(n)}
$$

where $\tilde{\mathbf{s}}$ and $\tilde{\mathbf{x}}_{0}$ are, respectively, the temporally whitened steering vector and test signal obtained with the true temporal correlation matrices $\mathbf{A}(p), p=1, \cdots, P$,

$$
\begin{aligned}
\tilde{\mathbf{s}}(n) & =\mathbf{s}(n)+\sum_{p=1}^{P} \mathbf{A}^{H}(p) \mathbf{s}(n-p), \\
\tilde{\mathbf{x}}_{0}(n) & =\mathbf{x}_{0}(n)+\sum_{p=1}^{P} \mathbf{A}^{H}(p) \mathbf{x}_{0}(n-p) .
\end{aligned}
$$

In the case of unknown $\mathbf{A}$ and $\mathbf{Q}$, the above PMF cannot be implemented and, therefore, we need to replace $\mathbf{A}$ and $\mathbf{Q}$ with their ML estimates under the persymmetric constraints of (5) and (6).

\section{B. Persymmetric ML Estimate of $\mathbf{Q}$}

The persymmetric ML estimates of $\mathbf{A}$ and $\mathbf{Q}$ are obtained from training signals only. According to the signal model, the joint likelihood function of training signals can be written as

$$
p\left(\mathbf{x}_{1}, \cdots, \mathbf{x}_{K} ; \mathbf{A}, \mathbf{Q}\right)=\left[\frac{1}{\pi^{J}|\mathbf{Q}|} e^{-\operatorname{tr}\left(\mathbf{Q}^{-1} \boldsymbol{\Gamma}_{0}\right)}\right]^{K(N-P)},
$$

where

$$
K(N-P) \boldsymbol{\Gamma}_{0}=\sum_{k=1}^{K} \sum_{n=P}^{N-1} \varepsilon_{k}(n) \varepsilon_{k}^{H}(n),
$$

with definitions

$$
\varepsilon_{k}(n)=\mathbf{x}_{k}(n)+\sum_{p=1}^{P} \mathbf{A}^{H}(p) \mathbf{x}_{k}(n-p) .
$$

Alternatively, $K(N-P) \boldsymbol{\Gamma}_{0}$ can be rewritten as

$$
K(N-P) \boldsymbol{\Gamma}_{0}=\hat{\mathbf{R}}_{x x}+\mathbf{A}^{H} \hat{\mathbf{R}}_{y x}+\hat{\mathbf{R}}_{y x}^{H} \mathbf{A}+\mathbf{A}^{H} \hat{\mathbf{R}}_{y y} \mathbf{A},
$$

where

$$
\begin{aligned}
& \mathbf{A} \triangleq\left[\mathbf{A}^{H}(1), \mathbf{A}^{H}(2), \cdots, \mathbf{A}^{H}(P)\right]^{H} \\
& \hat{\mathbf{R}}_{x x}=\sum_{k=1}^{K} \sum_{n=P}^{N-1} \mathbf{x}_{k}(n) \mathbf{x}_{k}^{H}(n), \\
& \hat{\mathbf{R}}_{y y}=\sum_{k=1}^{K} \sum_{n=P}^{N-1} \mathbf{y}_{k}(n) \mathbf{y}_{k}^{H}(n), \\
& \hat{\mathbf{R}}_{y x}=\sum_{k=1}^{K} \sum_{n=P}^{N-1} \mathbf{y}_{k}(n) \mathbf{x}_{k}^{H}(n),
\end{aligned}
$$

and $\mathbf{y}_{k}(n)$ is a regression vector of $\mathbf{x}_{k}(n): \mathbf{y}_{k}(n) \triangleq$ $\left[\mathbf{x}_{k}^{T}(n-1), \mathbf{x}_{k}^{T}(n-2), \cdots, \mathbf{x}_{k}^{T}(n-P)\right]^{T}$. By exploiting the persymmetric property of $\mathbf{Q}$, i.e., (5), we have

$$
\operatorname{tr}\left(\mathbf{Q}^{-1} \boldsymbol{\Gamma}_{0}\right)=\operatorname{tr}\left(\mathbf{Q}^{-1} \frac{\boldsymbol{\Gamma}_{0}+\mathbf{E} \boldsymbol{\Gamma}_{0}^{*} \mathbf{E}}{2}\right),
$$


which leads to

$$
\ln p \propto-\ln |\mathbf{Q}|-\frac{1}{2} \operatorname{tr}\left(\mathbf{Q}^{-1}\left[\boldsymbol{\Gamma}_{0}+\mathbf{E} \boldsymbol{\Gamma}_{0}^{*} \mathbf{E}\right]\right) .
$$

Taking the derivative of $\ln p$ with respect to $\mathbf{Q}$ and equating the results to zero lead to the persymmetric ML estimate of $\mathbf{Q}$

$$
\hat{\mathbf{Q}}_{\mathrm{PML}}=\frac{1}{2}\left(\boldsymbol{\Gamma}_{0}+\mathbf{E} \boldsymbol{\Gamma}_{0}^{*} \mathbf{E}\right) \text {. }
$$

As a result, $\ln p \propto-\ln \left|\frac{1}{2}\left(\boldsymbol{\Gamma}_{0}+\mathbf{E} \boldsymbol{\Gamma}_{0}^{*} \mathbf{E}\right)\right|$. Therefore, the persymmetric ML estimate of $\mathbf{A}$ is equivalent to minimizing the determinant of $K(N-P)\left(\boldsymbol{\Gamma}_{0}+\mathbf{E} \boldsymbol{\Gamma}_{0}^{*} \mathbf{E}\right) / 2$.

\section{Persymmetric ML Estimate of $\mathbf{A}$}

Recall that $\mathbf{A}$ of (14) is formed by stacking $\mathbf{A}(p)$ column-wise. From (6), A has the following property

$$
\mathbf{A}=\mathbf{E}_{B} \mathbf{A}^{*} \mathbf{E}
$$

where $\mathbf{E}_{B}=\mathbf{I}_{P} \otimes \mathbf{E}$ with $\mathbf{I}_{P}$ denoting a $P \times P$ identity matrix.

From (13), $K(N-P)\left(\boldsymbol{\Gamma}_{0}+\mathbf{E} \boldsymbol{\Gamma}_{0}^{*} \mathbf{E}\right)$ can be expressed as

$$
\begin{aligned}
& K(N-P)\left(\boldsymbol{\Gamma}_{0}+\mathbf{E} \Gamma_{0}^{*} \mathbf{E}\right) \stackrel{(a)}{=}\left[\hat{\mathbf{R}}_{x x}+\mathbf{E} \hat{\mathbf{R}}_{x x}^{*} \mathbf{E}\right] \\
& +\left[\mathbf{A}^{H} \hat{\mathbf{R}}_{y x}+\mathbf{E}\left(\mathbf{A}^{H} \mathbf{E}_{B} \mathbf{E}_{B} \hat{\mathbf{R}}_{y x}\right)^{*} \mathbf{E}\right] \\
& +\left[\hat{\mathbf{R}}_{y x}^{H} \mathbf{A}+\mathbf{E}\left(\hat{\mathbf{R}}_{y x}^{H} \mathbf{E}_{B} \mathbf{E}_{B} \mathbf{A}\right)^{*} \mathbf{E}\right] \\
& +\left[\mathbf{A}^{H} \hat{\mathbf{R}}_{y y} \mathbf{A}+\mathbf{E}\left(\mathbf{A}^{H} \mathbf{E}_{B} \mathbf{E}_{B} \hat{\mathbf{R}}_{y y} \mathbf{E}_{B} \mathbf{E}_{B} \mathbf{A}\right)^{*} \mathbf{E}\right] \\
& \stackrel{(b)}{=}\left[\hat{\mathbf{R}}_{x x}+\mathbf{E} \hat{\mathbf{R}}_{x x}^{*} \mathbf{E}\right]+\mathbf{A}^{H}\left[\hat{\mathbf{R}}_{y x}+\mathbf{E}_{B} \hat{\mathbf{R}}_{y x}^{*} \mathbf{E}\right] \\
& +\left[\hat{\mathbf{R}}_{y x}^{H}+\mathbf{E}\left(\hat{\mathbf{R}}_{y x}^{H}\right)^{*} \mathbf{E}_{B}\right] \mathbf{A}+\mathbf{A}^{H}\left[\hat{\mathbf{R}}_{y y}+\mathbf{E}_{B} \hat{\mathbf{R}}_{y y}^{*} \mathbf{E}_{B}\right] \mathbf{A},
\end{aligned}
$$

where (a) has used the fact that $\mathbf{E}_{B} \mathbf{E}_{B}=\mathbf{I}_{J P}$, and $(b)$ is due to (20) and $\mathbf{E}_{B}^{H}=\mathbf{E}_{B}$. Denote the persymmetric matrices:

$$
\begin{aligned}
& \hat{\mathbf{R}}_{x x, P}=\left(\hat{\mathbf{R}}_{x x}+\mathbf{E}^{*} \hat{\mathbf{R}}_{x x}^{*} \mathbf{E}\right) / 2, \\
& \hat{\mathbf{R}}_{y x, P}=\left(\hat{\mathbf{R}}_{y x}+\mathbf{E}_{B} \hat{\mathbf{R}}_{y x}^{*} \mathbf{E}\right) / 2, \\
& \hat{\mathbf{R}}_{y y, P}=\left(\hat{\mathbf{R}}_{y y}+\mathbf{E}_{B} \hat{\mathbf{R}}_{y y}^{*} \mathbf{E}_{B}\right) / 2 .
\end{aligned}
$$

As a result, $K(N-P)\left(\boldsymbol{\Gamma}_{0}+\mathbf{E} \boldsymbol{\Gamma}_{0}^{*} \mathbf{E}\right) / 2$ can be rewritten as

$$
\begin{aligned}
& K(N-P)\left(\boldsymbol{\Gamma}_{0}+\mathbf{E} \boldsymbol{\Gamma}_{0}^{*} \mathbf{E}\right) / 2 \\
= & \hat{\mathbf{R}}_{x x, P}+\mathbf{A}^{H} \hat{\mathbf{R}}_{y x, P}+\hat{\mathbf{R}}_{y x, P}^{H} \mathbf{A}+\mathbf{A}^{H} \hat{\mathbf{R}}_{y y, P} \mathbf{A} \\
= & \left(\mathbf{A}^{H}+\hat{\mathbf{R}}_{y x, P}^{H} \hat{\mathbf{R}}_{y y, P}^{-1}\right) \hat{\mathbf{R}}_{y y, P}\left(\mathbf{A}^{H}+\hat{\mathbf{R}}_{y x, P}^{H} \hat{\mathbf{R}}_{y y, P}^{-1}\right)^{H} \\
& +\left(\hat{\mathbf{R}}_{x x, P}-\hat{\mathbf{R}}_{y x, P}^{H} \hat{\mathbf{R}}_{y y, P}^{-1} \hat{\mathbf{R}}_{y x, P}\right) .
\end{aligned}
$$

Since $\hat{\mathbf{R}}_{y y, P}$ is nonnegative definite and the remaining term $\hat{\mathbf{R}}_{x x, P}-\hat{\mathbf{R}}_{y x, P}^{H} \hat{\mathbf{R}}_{y y, P}^{-1} \hat{\mathbf{R}}_{y x, P}$ does not depend on $\mathbf{A}$, it follows that

$$
\begin{aligned}
\left(\boldsymbol{\Gamma}_{0}+\mathbf{E} \boldsymbol{\Gamma}_{0}^{*} \mathbf{E}\right) / 2 \geq & \left(\boldsymbol{\Gamma}_{0}+\mathbf{E} \boldsymbol{\Gamma}_{0}^{*} \mathbf{E}\right) /\left.2\right|_{\mathbf{A}=-\hat{\mathbf{R}}_{y x, P}^{H} \hat{\mathbf{R}}_{y y, P}^{-1}} \\
& =\frac{\hat{\mathbf{R}}_{x x, P}-\hat{\mathbf{R}}_{y x, P}^{H} \hat{\mathbf{R}}_{y y, P}^{-1} \hat{\mathbf{R}}_{y x, P}}{K(N-P)},
\end{aligned}
$$

which implies that

$$
\hat{\mathbf{A}}_{\mathrm{PML}}=-\hat{\mathbf{R}}_{y y, P}^{-1} \hat{\mathbf{R}}_{y x, P},
$$

and the persymmetric ML estimate of $\mathbf{Q}$ of (19) reduces to

$$
\hat{\mathbf{Q}}_{\mathrm{PML}}=\frac{\hat{\mathbf{R}}_{x x, P}-\hat{\mathbf{R}}_{y x, P}^{H} \hat{\mathbf{R}}_{y y, P}^{-1} \hat{\mathbf{R}}_{y x, P}}{K(N-P)} .
$$

\section{Per-PAMF}

By replacing $\mathbf{A}$ and $\mathbf{Q}$ with the persymmetric ML estimates of $\mathbf{A}$ and $\mathbf{Q}$ in (8), we obtained the Per-PAMF as

$$
T_{\text {Per-PAMF }}=\frac{\left|\sum_{n=P}^{N-1} \hat{\tilde{\mathbf{s}}}_{P}^{H}(n) \hat{\mathbf{Q}}_{\mathrm{PML}}^{-1} \hat{\tilde{\mathbf{x}}}_{0, P}(n)\right|^{2}}{\sum_{n=P}^{N-1} \hat{\tilde{\mathbf{s}}}_{P}^{H}(n) \hat{\mathbf{Q}}_{\mathrm{PML}}^{-1} \hat{\tilde{\mathbf{s}}}_{P}(n)}{\underset{H}{H_{0}}}_{H_{1}}^{\gtrless} \gamma_{\text {Per-PAMF }}
$$

where $\gamma_{\text {Per-PAMF }}$ is a threshold subject to a preset probability of false alarm, and the temporally whitened steering vector $\hat{\tilde{\mathbf{s}}}_{P}$ and test signal $\hat{\tilde{\mathbf{x}}}_{0, P}$ are adaptively obtained, respectively, with the persymmetric ML estimate $\hat{\mathbf{A}}_{\mathrm{PML}}$ of (26):

$$
\begin{gathered}
\hat{\tilde{\mathbf{s}}}_{P}(n)=\mathbf{s}(n)+\sum_{p=1}^{P} \hat{\mathbf{A}}_{\mathrm{PML}}^{H}(p) \mathbf{s}(n-p), \\
\hat{\tilde{\mathbf{x}}}_{0, P}(n)=\mathbf{x}_{0}(n)+\sum_{p=1}^{P} \hat{\mathbf{A}}_{\mathrm{PML}}^{H}(p) \mathbf{x}_{0}(n-p) .
\end{gathered}
$$

From (28), it is seen that the Per-PAMF inherits the reduced computational complexity of the conventional PAMF by performing successively a temporal whitening followed by a spatial whitening, in contrast to the computationally intensive joint spatio-temporal whitening of the covariance matrix based approach (e.g., the AMF [5]). On the other hand, it further improves robustness of the parameter estimation by exploiting the underlying structure of the disturbance covariance matrix via the persymmetric ML estimates of the AR coefficient matrices $\mathbf{A}$ and the spatial covariance matrix $\mathbf{Q}$.

\section{E. Comparison with the conventional PAMF}

Compared with the conventional PAMF [7], the proposed PerPAMF shares the same test variable as shown in (28), except the underlying estimates of the unknown parameters $\mathbf{A}$ and $\mathbf{Q}$. The conventional PAMF uses the unconstrained ML estimate from training signals:

$$
\begin{aligned}
\hat{\mathbf{A}}_{\mathrm{ML}} & =-\hat{\mathbf{R}}_{y y}^{-1} \hat{\mathbf{R}}_{y x}, \\
\hat{\mathbf{Q}}_{\mathrm{ML}} & =\frac{\hat{\mathbf{R}}_{x x}-\hat{\mathbf{R}}_{y x}^{H} \hat{\mathbf{R}}_{y y}^{-1} \hat{\mathbf{R}}_{y x}}{K(N-P)} .
\end{aligned}
$$

Compared with the above ML estimates, it is noted that the persymmetric ML estimates of $\mathbf{A}$ and $\mathbf{Q}$ in (26) and (27) explicitly utilize the persymmetric properties through the operations of (21), (22) and (23).

\section{Performance Evaluation}

In this section, simulation results are provided to demonstrate the efficiency of the proposed Per-PAMF in the training-limited case, e.g., $K \ll J N$. The performance is evaluated with two datasets: 1) a synthesized $A R$ dataset, in which the disturbance signal $\mathbf{d}_{k}$ is generated as a multichannel second-order AR process $(P=2)$ with a AR coefficient $\mathbf{A}$ and a spatial covariance matrix $\mathbf{Q}$ satisfying (6) and (5); and 2) a physical clutter dataset, in which the disturbance is generated according to the clutter model used in [20]. The signalto-interference-plus-noise ratio (SINR) is defined as

$$
\operatorname{SINR}=|\alpha|^{2} \mathbf{s}^{H} \mathbf{R}^{-1} \mathbf{s},
$$

where $\mathbf{R}$ is the spatial-temporal covariance matrix. Regarding the synthesized dataset, $\mathbf{R}$ corresponds to the selected $\mathbf{A}$ and $\mathbf{Q}$. The simulated performance is obtained by using at least 10000 Monte Carlo trials for the probability of false alarm $P_{f}=0.01$. Performance comparisons are made among the non-parametric AMF [5], the nonparametric persymmetric AMF (Per-AMF) [18], the conventional 


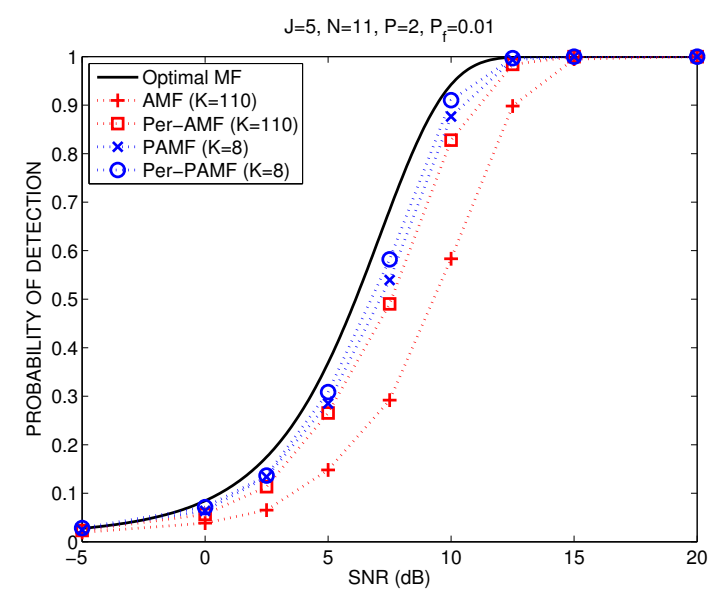

Fig. 1. Probability of detection versus SINR for $K=8$ when $J=5$, $N=11, P=2$, and $P_{f}=0.01$.

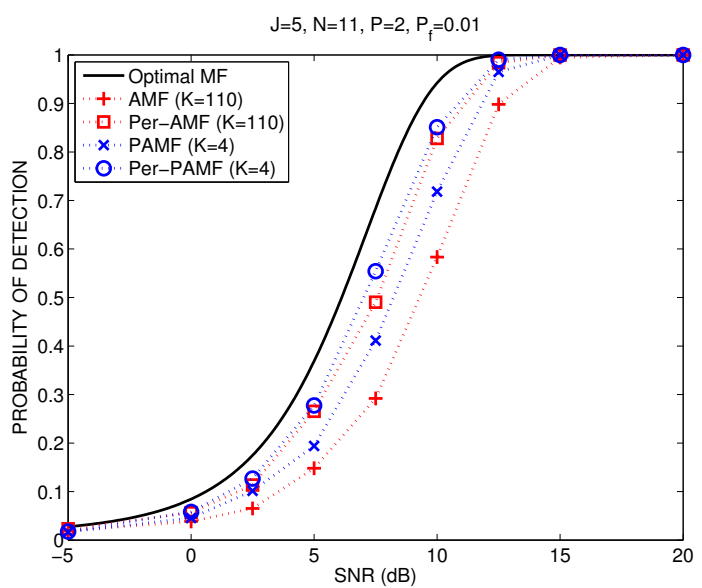

Fig. 2. Probability of detection versus SINR for $K=4$ when $J=5$, $N=11, P=2$, and $P_{f}=0.01$.

PAMF [7], and the clairvoyant matched filter (MF) [5], [20]. Particularly, the simulated scenario uses $J=5$ antenna elements and $N=11$ pulses, while the number of training signals are, respectively, $K=2, K=4$, and $K=8$.

\section{A. Synthesized AR Dataset}

In this case, the steering vector $\mathbf{s}$ is generated with a normalized spatial frequency $f_{s}=0.2$ and a normalized Doppler frequency is $f_{d}=0.2$, respectively. Fig. 1 shows the probability of detection versus the SINR with comparably sufficient training signals. In this case, the performance gain of the Per-PAMF over the conventional PAMF is marginal since both detectors have enough training signals to obtain good estimates of the unknown parameters. Meanwhile, both parametric detectors, i.e., the PAMF and Per-PAMF with $K=8$ training signals, show better detection performance than the nonparametric covariance matrix based AMF and Per-AMF with $K=$ $2 J N=110$ training signals.

In Fig. 2, the number of training signals is reduced to $K=4$. As shown in Fig. 2, the performance gap between the Per-PAMF and the PAMF is about $1.5 \mathrm{~dB}$ when $P_{d}=0.8$, while the Per-PAMF with $K=4$ training signals is slightly better than its nonparametric counterpart Per-AMF with $K=110$ training signals. The most challenging case is the third scenario where only $K=2$ training signals are available. As shown in Fig. 3, the conventional PAMF

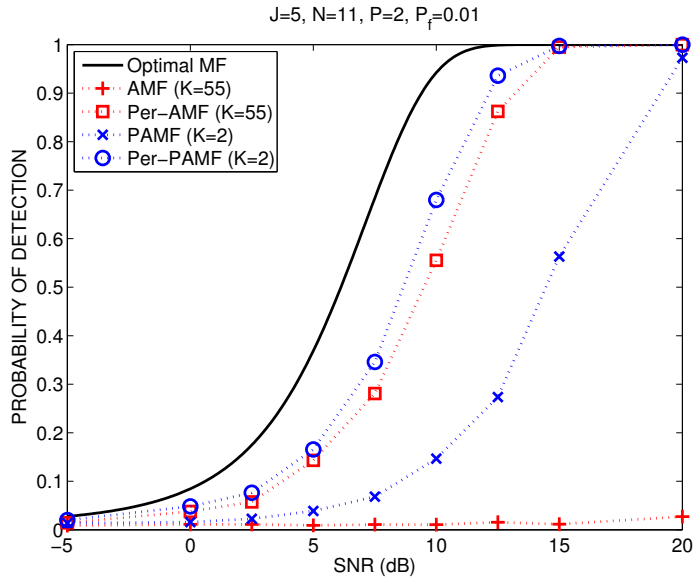

Fig. 3. Probability of detection versus SINR for $K=2$ when $J=5$, $N=11, P=2$, and $P_{f}=0.01$.

gives much worse performance than the Per-PAMF. In other words, with only $K=2$ training signals, the conventional PAMF cannot obtain reliable estimates of unknown parameters, e.g., $\mathbf{A}$ and $\mathbf{Q}$, which leads to performance degradation, while the Per-PAMF has better efficiency of using training signals for unknown parameter estimation and thus maintains its performance even with only $K=2$ training signals. The performance gain for the Per-PAMF over the conventional PAMF is about $5 \mathrm{~dB}$ when $P_{d}=0.8$. On the other hand, the AMF cannot work functionally with $K=J N=55$ training signals and the Per-AMF with $K=55$ training signals gives similar detection performance with the Per-PAMF with $K=2$ training signals.

From all three scenarios, it is clearly seen that, when there is a sufficient number of training signals, the performance gain is marginal and thus one may not need to utilize the prior persymmetric information. In contrast, the advantage of the proposed Per-PAMF becomes evident when the training signals are not enough for the PAMF to reliably estimate the unknown parameters and to maintain a reasonable detection performance. Therefore, in the training-limited scenarios, it is crucial to make use of the prior structure knowledge to improve the robustness of the parametric detectors. Here, we have a brief note on the CFAR property of the proposed Per-PAMF. Similar to the conventional PAMF, the CFAR property is usually achieved in the asymptotic case with a sufficiently large $N$ (the number of pulses) or at the sufficient training scenario where $K \gg J N$, but may vanish when $J \approx N$ or when the disturbance does not follow the AR process; see [Section VII.B] [7].

\section{B. Physical Clutter Dataset}

Unlike the previous section which used synthesized AR dataset, this section provides simulation results in a different environment, where the disturbance is generated from a physical clutter model described in [20], rather than a multichannel AR process. The platform is at altitude of $9 \mathrm{~km}$ with the velocity of $50 \mathrm{~m} / \mathrm{s}$, and the range of interest is $130 \mathrm{~km}$. The clutter-to-noise ratio (CNR) is $35 \mathrm{~dB}$. The clutter is divided among 360 clutter patches equally distributed in the azimuth about the platform and the RCS for each patch is weighted by a transmit pattern with a backlobe level of -30 $\mathrm{dB}$; see [20, eq. (80) and Figs. 9 and 10]. Moreover, we assume a linear symmetric array and a set of pulses with a constant PRF of $300 \mathrm{~Hz}$, which results in a persymmetric spatial steering vector and 


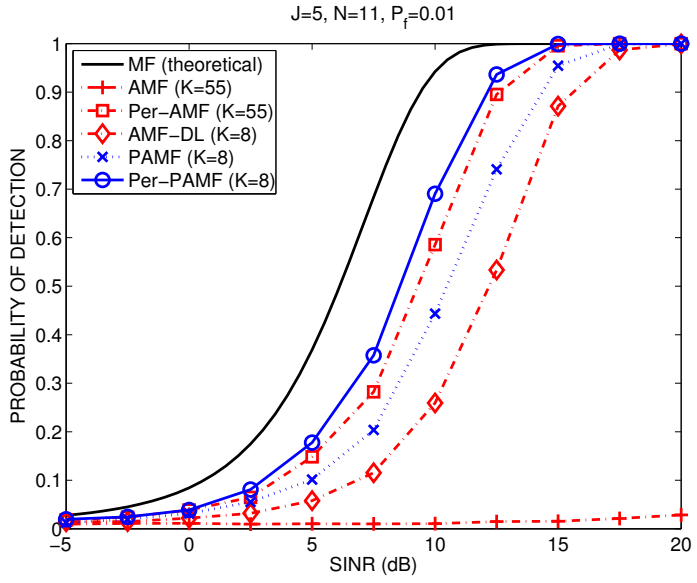

Fig. 4. Probability of detection versus SINR for $K=8$ when $J=5$, $N=11$, and $P_{f}=0.01$.

a Vandermonde temporal steering vector:

$$
\begin{aligned}
& \mathbf{s}_{s}=\left[e^{j 2 \pi 3 f_{s}}, e^{j 2 \pi f_{s}}, 1, e^{-j \pi f_{s}}, e^{-j 2 \pi 3 f_{s}}\right]^{T}, \\
& \mathbf{s}_{t}=\left[1, e^{j 2 \pi f_{d}}, e^{j 2 \pi 2 f_{d}}, \cdots, e^{j 2 \pi 9 f_{d}}, e^{j 2 \pi 10 f_{d}}\right]^{T} .
\end{aligned}
$$

The steering vectors for the clutter sources are similarly generated.

We consider the case with $K=8$ training signals. Since there is no knowledge about the AR order, we try a variety of AR orders, i.e., $P=\{1,2,3\}$, and select the one giving the best performance. In contrary, we use $K=55$ training signals for the non-parametric detectors, e.g., the conventional AMF and Per-AMF. Alternatively, the AR order can be adaptively determined from the training signals with conventional order determination techniques, e.g., the Akaike Information Criterion (AIC) and the minimum description length (MDL). Specifically, a low-complexity, joint model-order selection and parametric detection procedure was proposed in [21]. The simulated results are shown in Fig. 4. It is seen that the proposed PerPAMF is better than the conventional PAMF with a performance gain of $2 \mathrm{~dB}$ at $P_{d}=0.9$. Moreover, it is seen that the proposed Per-PAMF detector needs much less training signals ( $K=8$ versus $K=55)$ than the non-parametric Per-AMF detector to achieve similar detection performance, and is significantly better than the conventional AMF detector with $K=55$ training signals. We also note that, compared with the same case of $K=8$ but with the synthesized AR dataset in Fig. 1, the performance gap between the PAMF/Per-PAMF and the optimal matched filter is increased, which shows that the parametric PAMF/Per-PAMF detectors suffer from the model mismatch. Finally, we also compare the proposed Per-PAMF with the diagonal-loaded AMF [22], [23] with the same amount of training signals, i.e., $K=8$. It is seen from Fig. 4 that, with a choice of 10-dB loading factor (ten times the white noise level), the diagonal-loaded AMF (denoted as the AMF-DL) gives a detection performance, much better than that of the conventional AMF detector but still worse than that of the proposed Per-PAMF detector.

\section{CONCLUSION}

This paper extends the conventional PAMF by exploiting the structure properties of the disturbance covariance matrix, for widely used systems with a symmetric antennas geometry and pulses with a constant PRF. The developed Per-PAMF shares the same detection statistics as the conventional PAMF but utilizes the structure information through the estimation of the unknown AR coefficient matrices $\mathbf{A}$ and spatial covariance matrix $\mathbf{Q}$. Numerical results have verified that the proposed Per-PAMF gives better detection performance than the conventional PAMF as well as the covariance matrix based detectors when training signals are limited.

\section{APPENDIX}

In the following, we show that the persymmetric AR based disturbance in Section II has a persymmetric-block-Toeplitz spacetime covariance matrix. First, the space-time covariance matrix of an arbitrary multichannel AR process $\mathbf{x}(n)$ is defined by

$$
\begin{aligned}
& \mathbf{R}=E\left\{\left[\begin{array}{c}
\mathbf{x}(0) \\
\mathbf{x}(1) \\
\vdots \\
\mathbf{x}(N-1)
\end{array}\right]\left[\begin{array}{llll}
\mathbf{x}^{H}(0) & \mathbf{x}^{H}(1) & \cdots & \mathbf{x}^{H}(N-1)
\end{array}\right]\right\} \\
& =\left[\begin{array}{cccc}
\mathbf{R}(0) & \mathbf{R}(-1) & \cdots & \mathbf{R}(1-N) \\
\mathbf{R}(1) & \mathbf{R}(0) & \cdots & \mathbf{R}(2-N) \\
\vdots & \vdots & \ddots & \vdots \\
\mathbf{R}(N-1) & \mathbf{R}(N-2) & \cdots & \mathbf{R}(0)
\end{array}\right],
\end{aligned}
$$

where $\mathbf{R}(m)=E\left[\mathbf{x}(n) \mathbf{x}^{H}(n-m)\right]$ which implies that $\mathbf{R}(-m)=$ $\mathbf{R}^{H}(m)$. It is easily observed that $\mathbf{R}$ is a block-Toeplitz matrix.

To further show $\mathbf{R}$ is persymmetric-block-Toeplitz, we need to prove that the sub-block matrix $\mathbf{R}(m)$ is a persymmetric matrix. To this end, we consider an iterative procedure to find an explicit expression of $\mathbf{R}(m)$ as a function of the AR coefficient matrices $\mathbf{A}(p)$ and spatial covariance matrix $\mathbf{Q}$ and, then, prove the persymmetric property of the sub-block matrices $\mathbf{R}(m)$. First, we treat the AR process as a causal filter and $\mathbf{x}(n)$ is therefore the output of the causal filter

$$
\mathbf{x}(n)=\sum_{m=0}^{\infty} \mathbf{H}(m) \boldsymbol{\varepsilon}(n-m) .
$$

Given $\mathbf{H}(m)$, we have, for $m>0$

$$
\begin{aligned}
\mathbf{R}(m) & =E\left[\mathbf{x}(n) \mathbf{x}^{H}(n-m)\right] \\
& =E\left[\sum_{l_{1}=0}^{\infty} \mathbf{H}\left(l_{1}\right) \varepsilon\left(n-l_{1}\right) \sum_{l_{2}=0}^{\infty} \varepsilon^{H}\left(n-l_{2}\right) \mathbf{H}^{H}\left(l_{2}\right)\right] \\
& =\sum_{l_{1}=0}^{\infty} \sum_{l_{2}=0}^{\infty} \mathbf{H}\left(l_{1}\right) E\left[\varepsilon\left(n-l_{1}\right) \varepsilon^{H}\left(n-l_{2}\right)\right] \mathbf{H}^{H}\left(l_{2}\right) \\
& \stackrel{(a)}{=} \sum_{l_{1}=0}^{\infty} \sum_{l_{2}=0}^{\infty} \mathbf{H}\left(l_{1}\right) \mathbf{Q} \delta\left(m+l_{2}-l_{1}\right) \mathbf{H}^{H}\left(l_{2}\right) \\
& =\sum_{l_{1}=0}^{\infty} \mathbf{H}\left(l_{1}\right) \mathbf{Q} \mathbf{H}^{H}\left(l_{1}-m\right)
\end{aligned}
$$

where (a) holds since the driving noise $\varepsilon(n)$ is temporally white across $n$. From (35), it is seen that the output $\mathbf{x}(n)$ with respect to impulse input is

$$
\begin{aligned}
\mathbf{x}(n) & =\sum_{m=0}^{\infty} \mathbf{H}(m) \boldsymbol{\delta}(n-m) \\
& =\mathbf{H}(0) \boldsymbol{\delta}(n)+\mathbf{H}(1) \boldsymbol{\delta}(n-1)+\mathbf{H}(2) \boldsymbol{\delta}(n-2)+\cdots
\end{aligned}
$$

and we can enumerate

$$
\begin{aligned}
& \mathbf{x}(0)=\mathbf{H}(0) \boldsymbol{\delta}(0) \\
& \mathbf{x}(1)=\mathbf{H}(1) \boldsymbol{\delta}(0) \\
& \mathbf{x}(2)=\mathbf{H}(2) \boldsymbol{\delta}(0)
\end{aligned}
$$


and so on. Meanwhile, since $\mathbf{x}(n)$ is a multichannel AR process, we have

$$
\mathbf{x}(n)=-\sum_{p=1}^{P} \mathbf{A}^{H}(p) \mathbf{x}(n-p)+\varepsilon(n),
$$

and, therefore, the output $\mathbf{x}(n)$ with respect to impulse input can be alternatively obtained as

$$
\begin{aligned}
\mathbf{x}(0) & =\boldsymbol{\delta}(0), \\
\mathbf{x}(1) & =-\mathbf{A}^{H}(1) \mathbf{x}(0)=-\mathbf{A}^{H}(1) \boldsymbol{\delta}(0), \\
\mathbf{x}(2) & =-\mathbf{A}^{H}(1) \mathbf{x}(1)-\mathbf{A}^{H}(2) \mathbf{x}(0) \\
& =\left\{\left(-\mathbf{A}^{H}(1)\right)^{2}-\mathbf{A}^{H}(2)\right\} \boldsymbol{\delta}(0),
\end{aligned}
$$

and so on. By comparing (38) with (40), we have

$$
\begin{aligned}
& \mathbf{H}(0)=\mathbf{I}, \\
& \mathbf{H}(1)=-\mathbf{A}^{H}(1), \\
& \mathbf{H}(2)=-\mathbf{A}^{H}(1) \mathbf{H}(1)-\mathbf{A}^{H}(2) \mathbf{H}(0), \\
& \mathbf{H}(3)=-\mathbf{A}^{H}(1) \mathbf{H}(2)-\mathbf{A}^{H}(2) \mathbf{H}(1)-\mathbf{A}^{H}(3) \mathbf{H}(0),
\end{aligned}
$$

and so on. As a result, the impulse response matrix $\mathbf{H}(m)$ can be determined in an iterative way as

$$
\mathbf{H}(m)= \begin{cases}-\sum_{p=1}^{P} \mathbf{A}^{H}(p) \mathbf{H}(m-p), & m \geq P \\ -\sum_{p=1}^{m} \mathbf{A}^{H}(p) \mathbf{H}(m-p), & 0<m<P \\ \mathbf{I}, & m=0\end{cases}
$$

Since $\mathbf{A}(p), p=1, \cdots, P$, satisfy the persymmetric property as shown in (6), we can conclude from (42) that $\mathbf{H}(m)$ is also subject to the persymmetric constraint as

$$
\mathbf{H}(m)=\mathbf{E H}^{*}(m) \mathbf{E} .
$$

In addition to the spatial persymmetry of $\mathbf{Q}$ in (5), we can rewrite the block matrices $\mathbf{R}(m)$ in (36) as

$$
\begin{aligned}
\mathbf{E R}^{*}(m) \mathbf{E} & =\sum_{l_{1}=0}^{\infty} \mathbf{E H}^{*}\left(l_{1}\right) \mathbf{Q}^{*} \mathbf{H}^{T}\left(l_{1}-m\right) \mathbf{E} \\
& \stackrel{(a)}{=} \sum_{l_{1}=0}^{\infty}\left\{\mathbf{E} \mathbf{H}^{*}\left(l_{1}\right) \mathbf{E}\right\}\left\{\mathbf{E Q}^{*} \mathbf{E}\right\}\left\{\mathbf{E} \mathbf{H}^{T}\left(l_{1}-m\right) \mathbf{E}\right\} \\
& \stackrel{(b)}{=} \sum_{l_{1}=0}^{\infty} \mathbf{H}\left(l_{1}\right) \mathbf{Q} \mathbf{H}^{H}\left(l_{1}-m\right) \\
& =\mathbf{R}(m),
\end{aligned}
$$

where $(a)$ used the fact that $\mathbf{E E}=\mathbf{I}$ and $(b)$ holds due to the persymmetric properties of the $\mathbf{H}(m)$ and $\mathbf{Q}$. Combining (34) and (44), the overall space-time covariance matrix $\mathbf{R}$ of a persymmetric AR process is proved to be a persymmetric-block-Toeplitz matrix.

\section{REFERENCES}

[1] A. Paulraj and C. B. Papadias, "Space-time processing for wireless communications," IEEE Signal Processing Magazine, vol. 14, pp. 4983, November 1997.

[2] R. Klemm, Principles of Space-Time Adaptive Processing, The Institute of Electrical Engineers, London, UK, 2002.

[3] L. Huang, E. A. Thompson, V. Schmithorst, S. K. Holland, and T. M. Talavage, "Partially adaptive STAP algorithm approaches to functional MRI," IEEE Trans. Biomed. Eng., vol. 56, no. 2, pp. 518-521, February 2009.

[4] E. J. Kelly, "An adaptive detection algorithm," IEEE Transactions on Aerospace and Electronic Systems, vol. 22, pp. 115-127, March 1986.
[5] F. C. Robey, D. R. Fuhrmann, E. J. Kelly, and R. Nitzberg, "A CFAR adaptive matched filter detector," IEEE Transactions on Aerospace and Electronic Systems, vol. 28, no. 1, pp. 208-216, January 1992.

[6] A. De Maio, "Rao test for adaptive detection in Gaussian interference with unknown covariance matrix," IEEE Transactions on Signal Processing, vol. 55, no. 7, pp. 3577-3584, July 2007.

[7] J. R. Román, M. Rangaswamy, D. W. Davis, Q. Zhang, B. Himed, and J. H. Michels, "Parametric adaptive matched filter for airborne radar applications," IEEE Transactions on Aerospace and Electronic Systems, vol. 36, no. 2, pp. 677-692, April 2000.

[8] J. H. Michels, M. Rangaswamy, and B. Himed, "Performance of parametric and covariance based STAP tests in compound-Gaussian clutter," Digital Signal Processing, vol. 12, no. 2,3, pp. 307-328, April and July 2002.

[9] K. J. Sohn, H. Li, and B. Himed, "Parametric GLRT for multichannel adaptive signal detection," IEEE Transactions on Signal Processing, vol. 55, no. 11, pp. 5351-5360, November 2007.

[10] P. Wang, H. Li, and B. Himed, "A new parametric GLRT for multichannel adaptive signal detection," IEEE Trans. Signal Process., vol. 58, no. 1 , pp. 317-325, January 2010.

[11] P. Wang, H. Li, and B. Himed, "A Bayesian parametric test for multichannel adaptive signal detection in non-homogeneous environments," IEEE Signal Process. Lett., vol. 17, no. 4, pp. 351-354, April 2010.

[12] A. Cantoni and P. Butler, "Properties of the eigenvectors of persymmetric matrices with applications to communication theory," IEEE Trans. Commun., vol. 24, no. 8, pp. 804-809, August 1976.

[13] R Nitzberg, "Application of maximum likelihood estimation of persymmetric covariance matrices to adaptive processing," IEEE Trans. Aerosp. Electron. Syst., vol. 16, no. 1, pp. 124-127, January 1980.

[14] L. Cai and H. Wang, "A persymmetric multiband GLR algorithm," IEEE Trans. Aerosp. Electron. Syst., vol. 28, no. 3, pp. 806-816, July 1992.

[15] E. Conte and A. De Maio, "Exploiting persymmetry for CFAR detection in compound-Gaussian clutter," IEEE Trans. Aerosp. Electron. Syst., vol. 39, no. 2, pp. 719-724, April 2003.

[16] A. De Maio, G. Foglia, E. Conte, and A. Farina, "CFAR behavior of adaptive detectors: An experimental analysis," IEEE Trans. Aerosp. Electron. Syst., vol. 41, no. 1, pp. 233-251, January 2005.

[17] M. Casillo, A. De Maio, S. Iommelli, and L. Landi, "A persymmetric GLRT for adaptive detection in partially-homogeneous environment," IEEE Signal Process. Lett., vol. 14, no. 12, pp. 1016-1019, December 2007.

[18] G. Pailloux, P. Forster, J. P. Ovarlez, and F. Pascal, "On persymmetric covariance matrices in adaptive detection," in Proc. IEEE Int. Conf. on Acoust., Speech, and Signal Process., Las Vegas, Nevada, March/April 2008, pp. 2305-2308.

[19] M. Jansson, P. Wirfalt, K. Werner, and B. Ottersten, "ML estimation of covariance matrices with Kronecker and persymmetric structure," in Proc. IEEE DSP/SPE Workshop, Marco Island, FL, January 2009, pp. 298-301.

[20] J. Ward, "Space-time adaptive processing for airborne radar," Technical Report 1015, Lincoln Laboratory, MIT, December 1994.

[21] K. J. Sohn, H. Li, and B. Himed, "Recursive parametric tests for multichannel adaptive signal detection," IET Radar, Sonar and Navigation, vol. 2, no. 1, pp. 63-70, February 2008.

[22] Y. I. Abramovich, "Controlled method for adaptive optimization of filters using the criterion of maximum SNR," Radio Engineering and Electronic Physics, vol. 26, pp. 87-95, March 1981.

[23] B. D. Carlson, "Covariance matrix estimation errors and diagonal loading in adaptive arrays," IEEE Transactions on Aerospace and Electronic Systems, vol. 24, no. 4, pp. 397-401, July 1988. 